\title{
Quantum walk of two anyons across a statistical boundary
}

\author{
Liam L.H. Lau $\odot^{1, *, \dagger}$ and Shovan Dutta $\odot^{2, \ddagger, \S}$ \\ ${ }^{1}$ Gonville \& Caius College, University of Cambridge, Trinity Street, Cambridge CB2 1TA, United Kingdom \\ ${ }^{2}$ T.C.M. Group, Cavendish Laboratory, University of Cambridge, J.J. Thomson Avenue, Cambridge CB3 OHE, United Kingdom
}

(Received 17 August 2021; accepted 9 December 2021; published 31 January 2022)

\begin{abstract}
We model a quantum walk of identical particles that can alter their exchange statistics by hopping across a domain wall in a one-dimensional lattice. Such a "statistical boundary" is transparent to single particles and affects the dynamics only by swapping multiple particles arriving together. We find that the two-particle interference is dramatically altered by reflections of these bunched waves at the interface, producing strong measurable asymmetries. Depending on the phases on the two sides, a bunched wave packet can get completely reflected or split into a superposition of a reflected wave and an antibunched wave. This leads to striking dynamics with two domain walls, where bunched waves can get trapped in between or fragment into multiple correlated single-particle wave packets. These findings can be realized with density-dependent hopping in present-day atomic setups and open up a paradigm of intrinsically many-body phenomena at statistical boundaries.
\end{abstract}

DOI: 10.1103/PhysRevResearch.4.L012007

Introduction. The behavior of identical quantum particles is dictated by their exchange statistics, i.e., the phase $\theta$ acquired by the wave function when two particles are exchanged [1]. Bosons, with $\theta=0$, lead to blackbody radiation and Bose-Einstein condensates, whereas fermions, with $\theta=\pi$, form neutron stars and the periodic table of elements. More exotic particles, with $0<\theta<\pi$, can exist only in low dimensions [2-4]. These "anyons" are found as surface excitations on a fractional quantum Hall state [5] or spin liquids [6] and have been observed in recent experiments [7,8], but they can also arise in one dimension (1D) [9]. Their fractional statistics has fueled intense research [10-12] and is the basis for topological quantum computing protocols [13,14].

The rise of controllable atomic and photonic platforms has meant that one can engineer particle statistics in experiments [15,16]. In particular, Keilmann et al. [17] have shown that anyons on a $1 \mathrm{D}$ lattice are equivalent to bosons with densitydependent hopping, which has been realized in atomic setups [18-23]. Subsequently, Greschner and Santos [24] showed that the statistics of these anyons is fully tunable by a Raman laser. Motivated by such possibilities, theories have found rich ground states [25-27] and dynamics [28-30]. In these studies, the exchange phase, set by complex hopping amplitudes, is spatially uniform. Yet, the protocol in Ref. [24] can be extended to nonuniform phases, which produces an intriguing

\footnotetext{
*Present address: Rutgers University.

$\dagger$ liam.lh.lau@physics.rutgers.edu

‡Present address: Max Planck Institute for the Physics of Complex Systems.

§sdutta@pks.mpg.de

Published by the American Physical Society under the terms of the Creative Commons Attribution 4.0 International license. Further distribution of this work must maintain attribution to the author(s) and the published article's title, journal citation, and DOI.
}

scenario where anyons change their statistics by simply hopping across a domain wall. Here, we explore few-body physics resulting from such a "statistical boundary" by modeling twoparticle walks that can be monitored in experiments.

Two-body walks give a clean signature of the underlying statistics through the interference of multiple two-particle pathways, which produces bunching of bosons and antibunching of fermions, exemplified by the Hong-Ou-Mandel [31,32] effect. Such walks have been realized in atom traps [33-35] and photonic circuits [36-38], with key applications in quantum computing [39,40]. Two-body interference of anyons with a given statistics has also been examined [29,37,38,41]. In 1D models [17], the anyons can have double occupancies even at $\theta=\pi$ to allow for exchange, so in this limit they behave as "pseudofermions" that retain some bunching behavior [29].

We consider a statistical boundary where the exchange phase is $\alpha$ on one side and $\beta$ on the other side. On either side, the propagation occurs in the form of bunched and antibunched waves. Antibunched waves are unaffected by the interface since the two particles move separately. On the other hand, bunched waves are strongly transformed: For a boson-pseudofermion $(0-\pi)$ interface, we show that a bunched wave incident from the fermionic side is completely reflected, whereas one from the bosonic side is coherently split into a reflected bunched wave and an antibunched wave (Fig. 4). Hence the long-time dynamics are very sensitive to initial conditions, changing dramatically as one crosses the boundary. We fully characterize this physics by global number asymmetries that can be measured, e.g., with a quantum-gas microscope [35]. We predict striking consequences, including a statistical "well" that can trap or successively fragment bunched particles (Fig. 6). These features are most prominent for weak on-site interactions and large phase jumps at the boundary, which can both be tuned by Raman lasers [24].

Model. Anyons on a 1D lattice with a given exchange phase $\theta$ are defined by the commutation relations $\hat{a}_{j} \hat{a}_{k}=e^{i \theta} \hat{a}_{k} \hat{a}_{j}$ and 


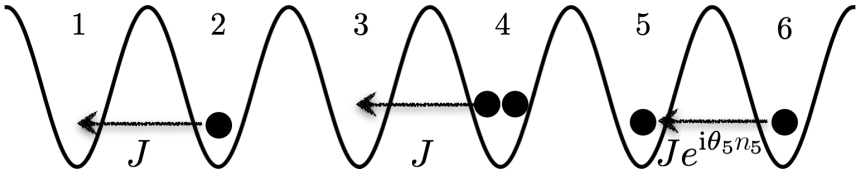

FIG. 1. Schematic of density-dependent tunneling in Eq. (4).

$\hat{a}_{j} \hat{a}_{k}^{\dagger}=e^{-i \theta} \hat{a}_{k}^{\dagger} \hat{a}_{j}$ for all $j<k$, where $\hat{a}_{j}^{\dagger}$ creates an anyon at site $j$. There is some freedom in choosing the on-site statistics $(j=k)$ [42]. We follow the convention in Refs. [17,43,44] where this is bosonic; i.e., $\left[\hat{a}_{j}, \hat{a}_{j}^{\dagger}\right]=1$, so multiple anyons can occupy the same site and exchange positions. This choice is further motivated by a Jordan-Wigner (JW) transform that maps such anyons to interacting bosons which can be studied experimentally [17].

We introduce anyons with a spatially varying exchange phase through a modified JW map,

$$
\hat{a}_{j}:=e^{i \sum_{k<j} \theta_{k} \hat{n}_{k}} \hat{b}_{j},
$$

where $\hat{b}_{j}$ are the boson operators and $\hat{n}_{k}:=\hat{b}_{k}^{\dagger} \hat{b}_{k}=\hat{a}_{k}^{\dagger} \hat{a}_{k}$ is the occupation at site $k$. Using the relations $\left[\hat{b}_{j}, \hat{b}_{k}\right]=0$ and $\left[\hat{b}_{j}, \hat{b}_{k}^{\dagger}\right]=\delta_{j k}$, we find the anyonic commutations

$$
\hat{a}_{j} \hat{a}_{k}=e^{i \theta_{j}} \hat{a}_{k} \hat{a}_{j} \quad \text { and } \quad \hat{a}_{j} \hat{a}_{k}^{\dagger}=e^{-i \theta_{j}} \hat{a}_{k}^{\dagger} \hat{a}_{j}
$$

for $j<k$; i.e., the exchange phase is set by the "left" site. This loss of reflection symmetry arises from Eq. (1) and is characteristic of anyons [10]. We are interested in cases where all exchanges are local and $\theta_{k}$ varies sharply across a domain wall, so particles on either side of the wall have well-defined and distinct statistics. Such an interface is "invisible" to single particles and affects the physics only by exchanging anyons between the two sides.

To probe the resulting dynamics, we adopt the anyonHubbard Hamiltonian [17]

$$
\hat{H}=-J \sum_{j}\left(\hat{a}_{j}^{\dagger} \hat{a}_{j+1}+\text { H.c. }\right)+U \sum_{j} \hat{n}_{j}\left(\hat{n}_{j}-1\right) / 2,
$$

where $J$ is the nearest-neighbor tunneling and $U$ is an on-site interaction. Crucially, this Hamiltonian maps onto a Hubbard model for bosons via Eq. (1),

$$
\hat{H}=-J \sum_{j}\left(\hat{b}_{j}^{\dagger} \hat{b}_{j+1} e^{i \theta_{j} \hat{n}_{j}}+\text { H.c. }\right)+\frac{U}{2} \sum_{j} \hat{n}_{j}\left(\hat{n}_{j}-1\right) .
$$

Here, $\theta_{j}$ gives an occupation-dependent Peierls phase; i.e., a hop from site $j+1$ to $j$ yields an additional phase depending on the occupation of site $j$, as shown in Fig. 1. Such phases have been realized in shaken optical lattices [19-22] in a quest to simulate dynamical gauge fields $[45,46]$. Furthermore, theoretical studies have shown that Peierls phases of the specific form in Eq. (4) can be engineered by Raman-assisted tunneling [17,24] or lattice shaking [47]. Of these, the protocol in Ref. [24] is particularly flexible and readily generalized to nonuniform $\theta_{j}$ by spatially modulating a Raman laser. Note that $\theta_{j}$ mediates an effective interaction between the bosons, which is distinct from the on-site interaction $U$. This is a consequence of the fractional anyonic statistics.

At any time $t$, the two-particle state can be expressed in terms of the boson operators as

$$
|\Psi(t)\rangle=\sum_{j} d_{j}(t) \hat{b}_{j}^{\dagger 2}|0\rangle / \sqrt{2}+\sum_{i<j} c_{i, j}(t) \hat{b}_{i}^{\dagger} \hat{b}_{j}^{\dagger}|0\rangle,
$$

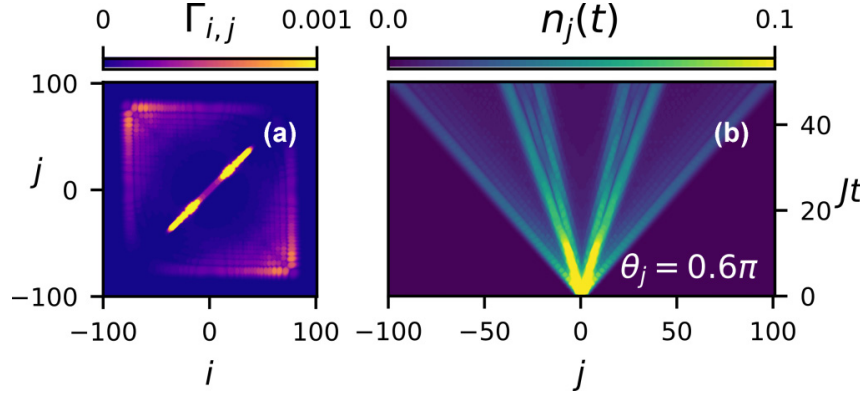

FIG. 2. Motion of two anyons with exchange phase $\theta_{j}=$ $0.6 \pi$ and on-site interaction $U=0$, starting from sites $j=0,1$. (a) Density-density correlations $\Gamma_{i, j}$ at time $t=40 / J$ and (b) density $n_{j}(t)$, showing fast antibunched and slow bunched waves.

where $|0\rangle$ is the vacuum and $\sum_{j}\left|d_{j}\right|^{2}+\sum_{i<j}\left|c_{i, j}\right|^{2}=1$ for normalization. Bunching or antibunching of the particles shows up in the density-density correlations $\Gamma_{i, j}:=\left\langle\hat{n}_{i} \hat{n}_{j}\right\rangle-$ $\delta_{i j} n_{i}=2\left|d_{j}\right|^{2} \delta_{i j}+\left|c_{i, j}\right|^{2}\left(1-\delta_{i j}\right)$ and can be measured experimentally [34-38]. Here, $n_{i}:=\left\langle\hat{n}_{i}\right\rangle=\sum_{j} \Gamma_{i, j}$. We focus on neighboring initial states where the anyons most strongly influence one another. We find the coefficients $d_{j}$ and $c_{i, j}$ by exact diagonalization, using a large grid to avoid reflection from the edges.

Uniform case. Before considering a domain wall, we discuss the physics in the uniform case [29], $\theta_{j}=\phi$, after the particles are released from adjacent sites. For $\phi=0$ and $U=0$, one has pure bosons that spread out in both directions as bunched waves with speed $2 J$ (in units of lattice spacing, with $\hbar=1$ ). For $\phi \neq 0$, one instead finds a superposition of bunched and antibunched propagation, as shown in Fig. 2. This can be seen either as a result of the anyonic statistics [Eq. (2)] or as a result of the occupation-dependent hopping of the JW bosons [Eq. (4)]. The antibunched wave describes the two particles moving in opposite directions at speed $2 J$, while the bunched wave is significantly slower. As $\phi$ is increased, the antibunching becomes more prominent, and the bunched waves slow down further. However, even in the pseudofermion limit, $\phi=\pi$, the latter carry almost half the total weight at a speed $v_{\text {slow }} \approx J / 5$ (see Supplemental Material [48]). One obtains stronger antibunching by increasing $U$. For $U / J \rightarrow \infty$, Eq. (4) reduces to hard-core bosons that behave like free fermions [35] regardless of $\phi$. So the exchange phase is more relevant at smaller $U$.

The slow bunched wave can be explained qualitatively for $\phi \lesssim 1$ by calculating the low-energy scattering length [24], which mimics an effective repulsion

$$
U_{\text {eff }}=4 J \tan ^{2}(\phi / 2)
$$

at $U=0$, leading to slow bound pairs [35]. However, this picture breaks down for large angles [49]. In particular, at $\phi=\pi$, $U_{\text {eff }} \rightarrow \infty$, which predicts free-fermionic behavior and does not support bunching.

Statistical boundary. We consider a sharp domain wall such that $\theta_{j}=\alpha$ for $j \leqslant 0$ and $\beta$ for $j>0$. We focus on a boson-pseudofermion interface, i.e., $\alpha=0$ and $\beta=\pi$, which produces the most striking departures. In a later section, we discuss how these effects fade gradually as one reduces the phase jump, increases $U$, or makes the interface less sharp. 


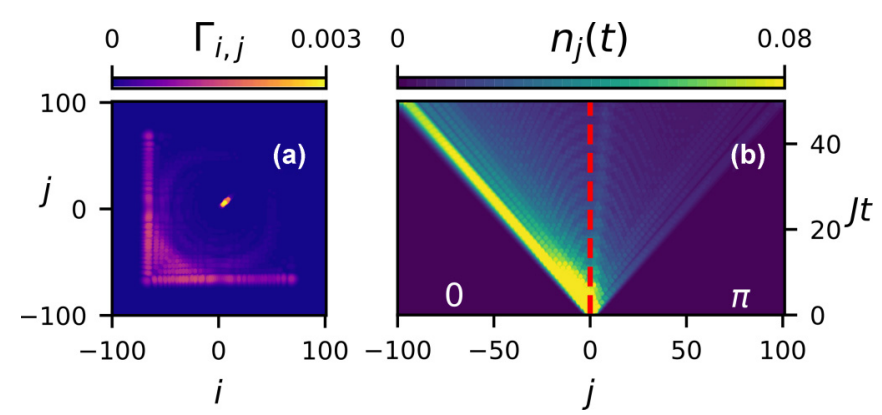

FIG. 3. Strongly asymmetric quantum walk of two anyons with $U=0$ in the presence of a boson-pseudofermion interface: $\theta_{j}=0$ for $j \leqslant 0$ and $\theta_{j}=\pi$ for $j>0$, after they are released from sites $j=$ 0,1 . (a) Two-body correlations at $J t=35$ and (b) density profile as a function of time, showing a pronounced bunched wave front on the bosonic side and a weak, slow bunched wave on the pseudofermion side.

Recall that $U$ and $\theta_{j}$ are both fully tunable by the protocol in Ref. [24].

Figure 3 shows what happens if the two particles are released from sites $j=0,1$, straddling the interface. An extremely skewed evolution ensues in which most of the weight flies off into the bosonic region as a bunched wave moving at speed $2 J$. This is accompanied by some remnant antibunching. The remainder is barely visible as a weak bunched wave moving into the pseudofermion side at speed $v_{\text {slow }}$, carrying less than $3 \%$ of the total weight. This is in stark contrast to the symmetric walk in Fig. 2. Here, the initial state can be considered "bosonic," as the first hop does not yield any Peierls phase (see Fig. 1), so we expect a bunched wave on the boson side. One might also anticipate less transmission to the pseudofermion region due to the effective repulsion [Eq. (6)]. However, as we pointed out before, this is only a qualitative picture. Below we show that the strong asymmetry originates from a characteristic reflection of the bunched waves off the domain wall.

To deconstruct this effect of the interface, we consider initial states farther away from it, so that the incident waves are clearly discernible. Figure 4(a) shows an example where the anyons are released well inside the bosonic region at $j=-6,-5$. As in the uniform case, they start spreading out as bunched waves in both directions. When the right-moving front arrives at the interface, we find that it is coherently split into two parts. One of these is reflected as a bunched wave, and the other turns into antibunched motion, where one particle enters the pseudofermion side and the other goes back to the boson side. This process is sketched in Fig. 4(b). Note that no bunched waves pass through the interface, which gives rise to the asymmetry in Fig. 3. The dynamics are even more striking when the anyons are released on the pseudofermion side. Here, one has two different timescales as in Fig. 2. There is a fast outward spreading where the anyons travel in opposite directions. Being solo, the left-moving anyon cannot see the interface and passes straight through, as in Fig. 4(c). Much later, the slow bunched wave arrives and gets completely reflected, as shown in Figs. 4(d) and 4(e).

The reflection of bunched waves incident from the boson side can be approximated by using effective hard-core
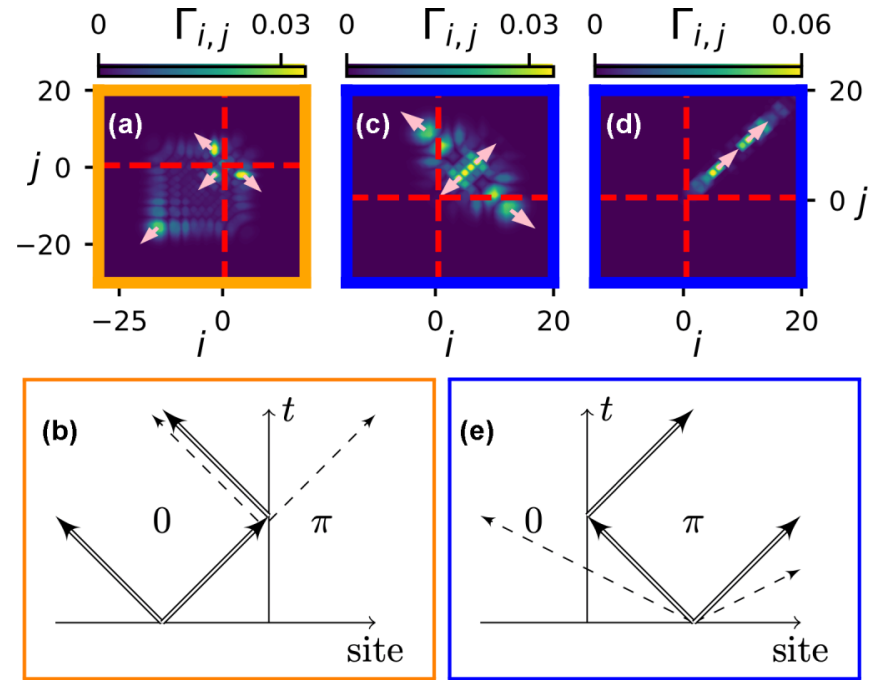

FIG. 4. Reflection of bunched waves off a $0-\pi$ statistical interface after two anyons with $U=0$ are released from (a)-(b) the boson side, $j=-6,-5$, and (c)-(e) the pseudofermion side, $j=5,6$. (a) Two-body correlations $\Gamma_{i, j}$ at $J t=5.9$, showing a bunched wave being split into a reflected bunched wave and an antibunched wave. (c) $\Gamma_{i, j}$ at $J t=4$, showing fast antibunched waves passing through the boundary and a slow bunched wave arriving at the interface. (d) $\Gamma_{i, j}$ at $J t=47.3$, showing that the slow bunched wave is completely reflected. (b) and (e) Sketch of the dynamics: double (single) arrows show bunched waves (single particles).

interactions for $j>0$, in accordance with Eq. (6). However, this recipe fails to capture the dynamics for pseudofermionic initial states (see Supplemental Material [48]). The lack of transmission of the bunched waves is consistent with a large difference in group velocity between the two sides. However, we emphasize that this is not single-particle physics, but a result of destructive interference between two-particle paths.

The dynamics are characterized by the weights in the bunched and antibunched waves, which can be extracted from the long-time distribution. In particular, since antibunched waves contain only one particle on each side, the weight in the bunched waves moving left (right) approximately equals the probability of finding both particles in the bosonic (fermionic) side, $P_{. .}^{b(f)}$. These are also related to the imbalance $\mathcal{I}:=\left(n^{b}-n^{f}\right) /\left(n^{b}+n^{f}\right)=P_{. .}^{b}-P_{. .}^{f}$, where $n^{b(f)}$ is the average number of particles on the boson (pseudofermion) side. As the initial positions cross the boundary, the physics changes drastically, producing sharp variations in $P_{\bullet \bullet}^{b(f)}$ and $\mathcal{I}$, as shown in Fig. 5. The asymmetry falls if the release sites are far inside the boson region since the bunching is not perfect and the particles have more time to delocalize. However, we do not see this behavior on the pseudofermion side, where the bunched motion is strongly bound. Similarly, $\mathcal{I}$ falls off with greater initial separation of the particles (see Supplemental Material [48]). Note that $P_{. .}^{b(f)}$ and $\mathcal{I}$ are directly measurable with a quantum-gas microscope [35].

Statistical well. The reflections sketched in Figs. 4(b) and 4(e) lead to striking dynamics when multiple domain walls coexist. Figure 6(a) shows a $\pi-0-\pi$ interface, where a bosonic region is sandwiched between two pseudofermion regions, 

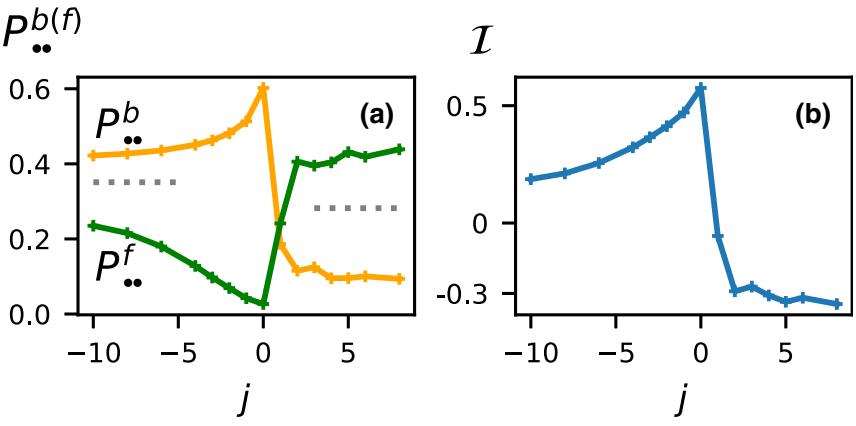

FIG. 5. Long-time asymmetries $(J t=100)$ after the anyons with $U=0$ are released from sites $j, j+1$, with the interface in Fig. 3 . (a) Probability of finding both particles in left (orange) and right (green) halves. Dotted lines show probabilities for uniform $\theta_{j}$ : $P_{. .}^{b(f)}\left(\theta_{j}=0\right) \approx 0.35$ and $P_{. \bullet}^{b(f)}\left(\theta_{j}=\pi\right) \approx 0.28$. (b) Relative number imbalance between the two halves. The sudden drop at $j=0$ signals very different physics on the two sides (see Fig. 4).

forming a "statistical well." Here, upon release in the middle, the particles repeatedly bounce back and forth as bunched waves, as per Fig. 4(b). At each bounce, nearly half the incoming flux leaks out into antibunched motion, giving rise to multiple correlated single-particle waves. The reflections are more prominent for a narrow boson region which reduces delocalization. On the other hand, for a $0-\pi-0$ interface, shown in Fig. 6(b), the loss and delocalization are both suppressed [as in Fig. 4(e)], but reflections occur on a longer timescale set by $v_{\text {slow }}$. In both cases, the width of the surrounding region is irrelevant as the bunched waves are confined inside the well; this is confirmed by numerics (see Supplemental Material [48]).

Experimental considerations. So far, we have considered zero on-site interactions and maximum phase jump across a sharp interface. In Fig. 7, we show that these conditions are by no means necessary for observing the physics. Figure 7(a) shows the imbalance $\mathcal{I}$ between two sides of a sharp 0- $\phi$ interface with $U \neq 0$, when the particles are released at the boundary (as in Fig. 3). As expected, $\mathcal{I}$ falls monotonically

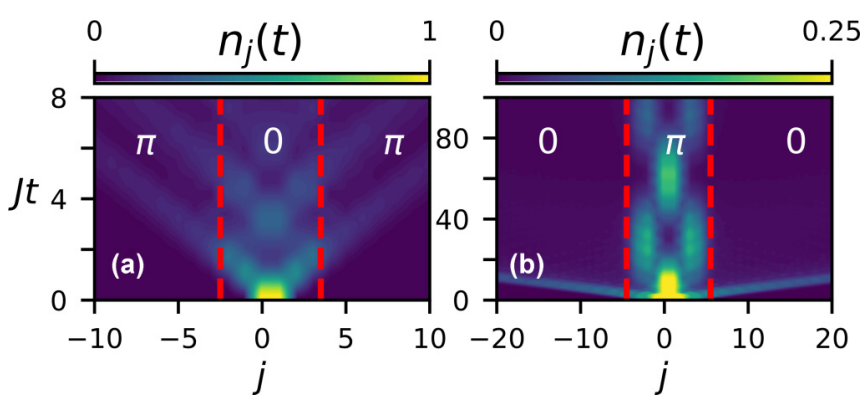

FIG. 6. Back-and-forth reflection of bunched waves inside a statistical well, after release of two anyons at $j=0,1$ with $U=0$. (a) $\pi-0-\pi$ interface: $\theta_{j}=0$ for $-2 \leqslant j \leqslant 3$. Reflections are lossy, as in Fig. 4(b), producing fragmented single-particle waves. (b) $0-\pi-0$ interface: $\theta_{j}=\pi$ for $-4 \leqslant j \leqslant 5$. Slow bunched waves undergo lossless reflections, and a fast antibunched wave front flies out at short times, as in Fig. 4(e).

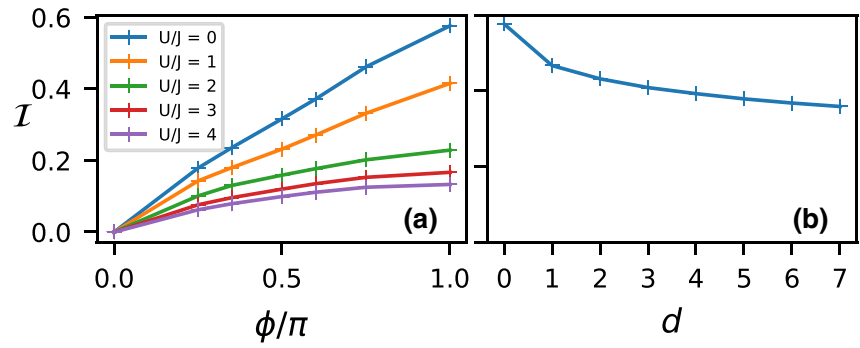

FIG. 7. (a) Long-time number imbalance between two sides of a $0-\phi$ boundary, $\theta_{j}=0$ for $j \leqslant 0$ and $\phi$ for $j>0$, with on-site interactions $U$, after two anyons are released from $j=0,1$. (b) Imbalance for a boundary of finite width $d$, over which $\theta_{j}$ varies from 0 to $\pi$, for the same initial state and $U=0$.

as $\phi$ is decreased or $U / J$ is increased. However, the change is gradual, and $\mathcal{I}$ remains large even for $U / J \sim 1$. In Fig. 7(b), we consider an interface of finite width $d$, where $\theta_{j}$ varies linearly from 0 to $\pi$ over $d$ sites. Here, $\mathcal{I}$ decreases slowly and reaches a plateau at large $d$, so the physics is also not sensitive to $d$.

As we stated earlier, of the several protocols for engineering anyon-Hubbard models $[12,17,24,47,50]$, the one in Ref. [24] is most suited for our purpose. Here, one uses a set of Raman lasers to control the tunneling of atoms with two internal states on a tilted optical lattice, such that $U$ is fully tunable by a detuning. The exchange statistics is set by the relative phase of one of the lasers, which can be varied spatially to form a domain wall.

Summary and outlook. We have investigated an intriguing scenario where multiple anyonic regions are separated by domain walls in the same physical system, and particles change their statistics by hopping across a wall. One can engineer this setting via occupation- and site-dependent hopping in realistic atomic setups. We have studied two-body walks in the vicinity of such a wall and showed that the dynamics are marked by a characteristic reflection of bunched waves at the interface that is strongly asymmetric and sensitive to initial conditions, leading to striking phenomena. These reflections leave experimentally measurable signatures in the long-time distribution.

A distinguishing feature of such a statistical interface is that it is, by definition, transparent to single particles and affects the physics only by exchanging multiple particles arriving simultaneously. Thus our findings strongly encourage future studies of this intrinsically many-body operation. For example, Refs. [17,24-26,50] have found insulating and superfluid phases of anyons as a function of their statistics, which can be combined to explore correlated transport through statistical junctions. Our work also highlights unexplored collective phenomena that open up by densitydependent gauge fields and motivate further experimental developments at this exciting frontier [19-23,45,46,51].

Acknowledgments. We thank Nigel Cooper for valuable feedback. This work was supported by Engineering and Physical Sciences Research Council Grant No. EP/P009565/1. 
[1] C. Cohen-Tannoudji, B. Diu, and F. Laloë, Quantum Mechanics (Wiley, London, 2006), Vol. 2.

[2] J. M. Leinaas and J. Myrheim, On the theory of identical particles, Nuovo Cimento Soc. Ital. Fis., B 37, 1 (1977).

[3] F. Wilczek, Quantum Mechanics of Fractional-Spin Particles, Phys. Rev. Lett. 49, 957 (1982).

[4] G. S. Canright and S. M. Girvin, Fractional statistics: Quantum possibilities in two dimensions, Science 247, 1197 (1990).

[5] A. Stern, Anyons and the quantum Hall effect - a pedagogical review, Ann. Phys. (Amsterdam) 323, 204 (2008).

[6] H. Yao and S. A. Kivelson, Exact Chiral Spin Liquid with NonAbelian Anyons, Phys. Rev. Lett. 99, 247203 (2007).

[7] H. Bartolomei, M. Kumar, R. Bisognin, A. Marguerite, J.-M. Berroir, E. Bocquillon, B. Plaçais, A. Cavanna, Q. Dong, U. Gennser, Y. Jin, and G. Fève, Fractional statistics in anyon collisions, Science 368, 173 (2020).

[8] J. Nakamura, S. Liang, G. C. Gardner, and M. J. Manfra, Direct observation of anyonic braiding statistics, Nat. Phys. 16, 931 (2020).

[9] F. D. M. Haldane, "Fractional Statistics" in Arbitrary Dimensions: A Generalization of the Pauli Principle, Phys. Rev. Lett. 67, 937 (1991).

[10] F. Wilczek, Fractional Statistics and Anyon Superconductivity (World Scientific, Singapore, 1990), Vol. 5.

[11] A. Kitaev, Anyons in an exactly solved model and beyond, Ann. Phys. (Amsterdam) 321, 2 (2006).

[12] S. Greschner, L. Cardarelli, and L. Santos, Probing the exchange statistics of one-dimensional anyon models, Phys. Rev. A 97, 053605 (2018).

[13] C. Nayak, S. H. Simon, A. Stern, M. Freedman, and S. D. Sarma, Non-Abelian anyons and topological quantum computation, Rev. Mod. Phys. 80, 1083 (2008).

[14] V. Lahtinen and J. Pachos, A short introduction to topological quantum computation, SciPost Phys. 3, 021 (2017).

[15] I. M. Georgescu, S. Ashhab, and F. Nori, Quantum simulation, Rev. Mod. Phys. 86, 153 (2014).

[16] E. Altman, K. R. Brown, G. Carleo, L. D. Carr, E. Demler, C. Chin, B. DeMarco, S. E. Economou, M. A. Eriksson, K.-M. C. Fu, M. Greiner, K. R. A. Hazzard, R. G. Hulet, A. J. Kollar, B. L. Lev, M. D. Lukin, R. Ma, X. Mi, S. Misra, C. Monroe et al., Quantum simulators: Architectures and opportunities, PRX Quantum 2, 017003 (2021).

[17] T. Keilmann, S. Lanzmich, I. McCulloch, and M. Roncaglia, Statistically induced phase transitions and anyons in 1D optical lattices, Nat. Commun. 2, 361 (2011).

[18] O. Jürgensen, F. Meinert, M. J. Mark, H.-C. Nägerl, and D.-S. Lühmann, Observation of Density-Induced Tunneling, Phys. Rev. Lett. 113, 193003 (2014).

[19] F. Meinert, M. J. Mark, K. Lauber, A. J. Daley, and H.-C. Nägerl, Floquet Engineering of Correlated Tunneling in the Bose-Hubbard Model with Ultracold Atoms, Phys. Rev. Lett. 116, 205301 (2016).

[20] L. W. Clark, B. M. Anderson, L. Feng, A. Gaj, K. Levin, and C. Chin, Observation of Density-Dependent Gauge Fields in a Bose-Einstein Condensate Based on Micromotion Control in a Shaken Two-Dimensional Lattice, Phys. Rev. Lett. 121, 030402 (2018).

[21] F. Görg, K. Sandholzer, J. Minguzzi, R. Desbuquois, M. Messer, and T. Esslinger, Realization of density-dependent
Peierls phases to engineer quantized gauge fields coupled to ultracold matter, Nat. Phys. 15, 1161 (2019).

[22] C. Schweizer, F. Grusdt, M. Berngruber, L. Barbiero, E. Demler, N. Goldman, I. Bloch, and M. Aidelsburger, Floquet approach to $\mathbb{Z}_{2}$ lattice gauge theories with ultracold atoms in optical lattices, Nat. Phys. 15, 1168 (2019).

[23] V. Lienhard, P. Scholl, S. Weber, D. Barredo, S. de Léséleuc, R. Bai, N. Lang, M. Fleischhauer, H. P. Büchler, T. Lahaye, and A. Browaeys, Realization of a Density-Dependent Peierls Phase in a Synthetic, Spin-Orbit Coupled Rydberg System, Phys. Rev. X 10, 021031 (2020).

[24] S. Greschner and L. Santos, Anyon Hubbard Model in OneDimensional Optical Lattices, Phys. Rev. Lett. 115, 053002 (2015).

[25] J. Arcila-Forero, R. Franco, and J. Silva-Valencia, Critical points of the anyon-Hubbard model, Phys. Rev. A 94, 013611 (2016).

[26] F. Lange, S. Ejima, and H. Fehske, Anyonic Haldane Insulator in One Dimension, Phys. Rev. Lett. 118, 120401 (2017).

[27] G. Tang, S. Eggert, and A. Pelster, Ground-state properties of anyons in a one-dimensional lattice, New J. Phys. 17, 123016 (2015).

[28] Y. Hao and S. Chen, Dynamical properties of hard-core anyons in one-dimensional optical lattices, Phys. Rev. A 86, 043631 (2012).

[29] L. Wang, L. Wang, and Y. Zhang, Quantum walks of two interacting anyons in one-dimensional optical lattices, Phys. Rev. A 90, 063618 (2014)

[30] L. Piroli and P. Calabrese, Exact dynamics following an interaction quench in a one-dimensional anyonic gas, Phys. Rev. A 96, 023611 (2017).

[31] C. K. Hong, Z. Y. Ou, and L. Mandel, Measurement of Subpicosecond Time Intervals between Two Photons by Interference, Phys. Rev. Lett. 59, 2044 (1987).

[32] A. M. Kaufman, M. C. Tichy, F. Mintert, A. M. Rey, and C. A. Regal, The Hong-Ou-Mandel effect with atoms, Adv. At., Mol., Opt. Phys. 67, 377 (2018).

[33] T. Jeltes, J. M. McNamara, W. Hogervorst, W. Vassen, V. Krachmalnicoff, M. Schellekens, A. Perrin, H. Chang, D. Boiron, A. Aspect, and C. I. Westbrook, Comparison of the Hanbury Brown-Twiss effect for bosons and fermions, Nature (London) 445, 402 (2007).

[34] M. Schellekens, R. Hoppeler, A. Perrin, J. V. Gomes, D. Boiron, A. Aspect, and C. I. Westbrook, Hanbury Brown Twiss effect for ultracold quantum gases, Science 310, 648 (2005).

[35] P. M. Preiss, R. Ma, M. E. Tai, A. Lukin, M. Rispoli, P. Zupancic, Y. Lahini, R. Islam, and M. Greiner, Strongly correlated quantum walks in optical lattices, Science 347, 1229 (2015).

[36] A. P. Peruzzo, M. Lobino, J. C. F. Matthews, N. Matsuda, A. Politi, K. Poulios, X.-Q. Zhou, Y. Lahini, N. Ismail, K. Wörhoff, Y. Bromberg, Y. Silberberg, M. G. Thompson, and J. L. OBrien, Quantum walks of correlated photons, Science 329, 1500 (2010).

[37] L. Sansoni, F. Sciarrino, G. Vallone, P. Mataloni, A. Crespi, R. Ramponi, and R. Osellame, Two-Particle Bosonic-Fermionic Quantum Walk via Integrated Photonics, Phys. Rev. Lett. 108, 010502 (2012). 
[38] J. C. F. Matthews, K. Poulios, J. D. A. Meinecke, A. Politi, A. Peruzzo, N. Ismail, K. Wörhoff, M. G. Thompson, and J. L. O'Brien, Observing fermionic statistics with photons in arbitrary processes, Sci. Rep. 3, 1539 (2013).

[39] A. M. Childs, Universal Computation by Quantum Walk, Phys. Rev. Lett. 102, 180501 (2009).

[40] S. E. Venegas-Andraca, Quantum walks: a comprehensive review, Quantum Inf. Process. 11, 1015 (2012).

[41] G. Campagnano, O. Zilberberg, I. V. Gornyi, D. E. Feldman, A. C. Potter, and Y. Gefen, Hanbury Brown-Twiss Interference of Anyons, Phys. Rev. Lett. 109, 106802 (2012).

[42] M. D. Girardeau, Anyon-Fermion Mapping and Applications to Ultracold Gases in Tight Waveguides, Phys. Rev. Lett. 97, 100402 (2006).

[43] A. Kundu, Exact Solution of Double $\delta$ Function Bose Gas through an Interacting Anyon Gas, Phys. Rev. Lett. 83, 1275 (1999).

[44] M. Bonkhoff, K. Jägering, S. Eggert, A. Pelster, M. Thorwart, and T. Posske, Bosonic Continuum Theory of One-Dimensional Lattice Anyons, Phys. Rev. Lett. 126, 163201 (2021).

[45] M. C. Bañuls, R. Blatt, J. Catani, A. Celi, J. I. Cirac, M. Dalmonte, L. Fallani, K. Jansen, M. Lewenstein, S. Montangero, C. A. Muschik, B. Reznik, E. Rico, L. Tagliacozzo, K. Van Acoleyen, F. Verstraete, U.-J. Wiese, M. Wingate, J. Zakrzewski, and P. Zoller, Simulating lattice gauge theories within quantum technologies, Eur. Phys. J. D 74, 165 (2020).
[46] M. Aidelsburger, L. Barbiero, A. Bermudez, T. Chanda, A. Dauphin, D. González-Cuadra, P. R. Grzybowski, S. Hands, F. Jendrzejewski, J. Jünemann, G. Juzeliūnas, V. Kasper, A. Piga, S.-J. Ran, M. Rizzi, G. Sierra, L. Tagliacozzo, E. Tirrito, T. V. Zache, J. Zakrzewski, E. Zohar, and M. Lewenstein, Cold atoms meet lattice gauge theory, Philos. Transact. Royal Soc. A 380, 20210064 (2021).

[47] C. Sträter, S. C. L. Srivastava, and A. Eckardt, Floquet Realization and Signatures of One-Dimensional Anyons in an Optical Lattice, Phys. Rev. Lett. 117, 205303 (2016).

[48] See Supplemental Material at http://link.aps.org/supplemental/ 10.1103/PhysRevResearch.4.L012007 for characterizations of the slow bunched waves, deviations from the effective-repulsion picture, dynamics of particles coincident from opposite directions with different initial separations, and insensitivity to the width of statistical regions surrounding a well.

[49] A. K. Kolezhuk, F. Heidrich-Meisner, S. Greschner, and T. Vekua, Frustrated spin chains in strong magnetic field: Dilute two-component Bose gas regime, Phys. Rev. B 85, 064420 (2012).

[50] L. Cardarelli, S. Greschner, and L. Santos, Engineering interactions and anyon statistics by multicolor lattice-depth modulations, Phys. Rev. A 94, 023615 (2016).

[51] K.-X. Yao, Z. Zhang, and C. Chin, Dynamics of domain walls in a Bose-Einstein condensate driven by density-dependent gauge field, arXiv:2105.15052. 